\title{
Monte Carlo Simulations of the Influence of Localization Centres on Carrier Dynamics in GaInNAs Quantum Wells
}

\author{
M. Baranowski*, R. Kudrawiec, M. Latkowska, M. Syperek and J. Misiewicz \\ Institute of Physics, Wrocław University of Technology, Wybrzeże Wyspiańskiego 27, 50-370 Wrocław, Poland

\begin{abstract}
Model of hopping excitons is applied to study the carrier dynamics in GaInNAs/GaAs quantum well system. Impact of parameters describing localizing states (i.e., an average energy and density) on carrier dynamics in GaInNAs material is investigated theoretically. It is shown how those parameters affect the quantities that can be extracted from time resolved photoluminescence experiments. It is shown that obtained simulations can be very helpful in the interpretation of the experimental data.
\end{abstract}

PACS: 78.55.Cr, 78.20.Bh, 78.55.Qr

\section{Introduction}

The GaInNAs alloys since many years have been under the great interest of scientists due to their potential application in optoelectronic devices. The incorporation of small amount of nitrogen into GaInAs causes significant reduction of the bandgap energy with the simultaneous decrease of the lattice constant. Those features make GaInNAs very attractive for use in telecommunication lasers [1] and solar cells based on GaAs [2]. The incorporation of nitrogen into GaInAs host also strongly affects the photoluminescence (PL) from this material system. The PL spectra become broader and asymmetric after the addition of nitrogen. Temperature dependence of PL peak energy exhibits deviation from Varshni's formula, i.e. so called S-shape $[3,4]$ and PL decay times show strong spectral dispersion at low temperatures [5]. Additionally we can observe in micro-PL experiment that the broad PL band observed at low temperature is composed of sharp lines $[6,7]$, which are associated with the recombination of single excitons trapped by the localization potentials induced by the nitrogen incorporation.

The characteristic features observed in PL experiments can be described by the model of hopping excitons [8] which refers to the dynamics of excitons in the disordered semiconductor compounds. It was successfully applied to explain phenomena observed in macro-PL spectra in diluted nitrides $[8,9]$. Recently we have shown that this model with some modifications can be also applied to explain the origin of sharp lines observed in micro-PL experiment [10] and can excellently reproduce the PL dynamics for GaInNAs/GaAs quantum well (QW) [11].

In this work we use model of hopping excitons to study the influence of localizing states on excitons and PL dy-

* corresponding author; e-mail: michal.baranowski@pwr.wroc.pl namics in GaInNAs alloy. We show how the changes of localizing states density and energy affect the characteristic features of PL dynamics that can be observed in time resolved experiment.

\section{Theoretical model}

We carried out Monte Carlo simulations of PL dynamics including different scenarios leading to exciton recombination. The simulation is in detail described in the previous works $[10,11]$, so here we just present a brief description. In this model it is assumed that excitons behave like single non-interacting particles. The rates (probabilities) of different processes are described by formulae presented below. Our model takes into account recombination from localized states as well as from delocalized states. The rate of radiative recombination is equal

$$
v_{\mathrm{r}}=\tau_{0}^{-1},
$$

where $\tau_{0}$ is the exciton life time. The rate of exciton activation above the mobility edge from a localized state $i$ with the energy $\varepsilon_{i}$ below the mobility edge is equal to

$$
v_{\mathrm{a}}=v_{0} \exp \left(-\frac{\varepsilon_{i}}{k T}\right)
$$

and the hopping rate from side $i$ to unoccupied side $j$ is calculated according to the Miller-Abrahams formula

$$
v_{i j}=v_{0} \exp \left(-\frac{2 r_{i j}}{\alpha}-\frac{\varepsilon_{j}-\varepsilon_{i}+\left|\varepsilon_{j}-\varepsilon_{i}\right|}{2 k T}\right),
$$

where $\varepsilon_{i}$ is the energy of localized state, $\alpha$ is the decay length of exciton wave function and $v_{0}$ is the attempt to escape frequency. We assume that the rate of nonradiative recombination is described by the expression

$$
v_{\mathrm{nr}}=v_{\mathrm{d}} \exp \left(\frac{-\varepsilon_{\mathrm{b}}}{k T}\right),
$$

where the $v_{\mathrm{d}}$ is the rate of exciton dissociation (nonradiatve recombination) and the $\varepsilon_{\mathrm{b}}$ is the binding energy of 
free excitons in GaInNAs/GaAs QW. A free exciton can be captured by one of unoccupied localized centres with the rate

$$
v_{\text {cap }}=v_{0} \frac{N_{\mathrm{f}}}{N},
$$

where $N_{\mathrm{f}}$ is a number of unoccupied localized states and $N$ is the total number of localized states. Proposed model takes into account saturation effects and simulations can be performed with different number of excitons created at the beginning. Free exciton recombines with some random energy which is described by the Gaussian distribution resulting from alloy inhomogeneities. For recombination from localized states we use the Lorentz line shape and we assume an exponential density of localized states (DOS)

$$
\operatorname{DOS}(\varepsilon)=\frac{N / L^{2}}{\varepsilon_{0}} \exp \left(-\frac{\varepsilon}{\varepsilon_{0}}\right),
$$

where $N$ is the number of defect states, $L$ is the size of $2 \mathrm{D}$ volume where defect states are situated in a random way and $\varepsilon_{0}$ is an average value of energy of localizing states. In this paper we investigate the influence of parameters $\frac{N \alpha^{2}}{L^{2}}$ and $\varepsilon_{0}$ on carrier dynamics in GaInNAs/GaAs QW system. The $\frac{N \alpha^{2}}{L^{2}}$ parameter can be treated as a density of localizing states (its value determines the hopping efficiency between different localizing states) while $\varepsilon_{0}$ represents the deepness of localizing potential.

The simulation algorithm is the following. We create a list which contains a given number $\left(n_{0}\right)$ of free excitons at the time $t=0$. Next we take the first exciton from the list and determine the type of process for this exciton and the time for the next process for this exciton. After this procedure excitons are sorted on the list according to the time of the next process described by equation

$$
t_{i}=-\frac{1}{v_{i} \ln \theta},
$$

where $\theta$ is a random number taken from the uniform distribution between 0 and 1 and the total decay rate $v_{i}$ at a given localization centre is calculated as

$$
v_{i}=v_{\mathrm{r}}+v_{\mathrm{a}}+\sum_{i \neq j} v_{i j} .
$$

Exciton is removed from the list if the recombination process took place and the time and energy of radiative recombination is recorded. This procedure is repeated until all excitons recombine.

\section{Results}

Simulations were performed with parameters' values typical for GaInNAs/GaAs QW system, i.e., $v_{0}=$ $10^{13} \mathrm{~s}^{-1}, \tau_{0}=0.83 \mathrm{~ns}, \tau_{0} v_{\mathrm{d}} \approx 27$ and $\varepsilon_{\mathrm{b}} \approx 8 \mathrm{meV}$ [8, 10-13]. Gaussian and Lorentzian line broadening are $7 \mathrm{meV}$ and $0.7 \mathrm{meV}$, respectively. Number of defects is $N=2000$ and temperature $T$ equals $10 \mathrm{~K}$. In real time resolved PL (TRPL) measurements sample is excited by a pulsed laser which results in a partial saturation of localizing states. To imitate excitation conditions typical for TRPL measurements, the initial number of injected carriers in our simulations equals $n_{0}=1000$ [11]. In this excitation regime most of the carriers recombine via localizing states and free excitons recombination is negligibly small. Simulations are performed for different $\frac{N \alpha^{2}}{L^{2}}$ and $\varepsilon_{0}$ value to show the influence of these parameters on PL dynamics. Simulations are repeated many times in order to obtain reliable results.

Figure 1 shows some exemplary decay curves for different energies below the band gap (i.e., the mobility edge). It is clearly visible that the decay becomes longer with the decrease in emission energy. This observation is connected with the phenomenon of exciton hopping. The time decay at higher energies is reduced because the exciton recombination dynamics is affected by the energy transfer process to states with lower energies. Simultaneously the exciton transfer from low energy states to high energy states is damped since excitons do not have sufficient thermal energy for such a transfer. Due to this asymmetry of hopping rates between low and high energy states the PL decay is elongated at the low energy side due to refilling the states by relaxing excitons. In order to perform a detailed analysis of this phenomenon, the PL decay curves have been fitted by the single exponential function $I(t)=I_{0} \exp \left(-t / \tau_{\mathrm{PL}}\right)$, where $t$ is the elapsed time, $I_{0}$ is the PL signal intensity just after the excitation pulse, and $\tau_{\mathrm{PL}}$ is the PL decay time. In most cases the PL decay curve can be quite well fitted by the single exponential formula (see red lines in Fig. 1b and c). However, for some energies the decays are not exponential and then for the simplicity and clarity of performed analysis we fit only initial part of the curve as it is shown in Fig. 1a.

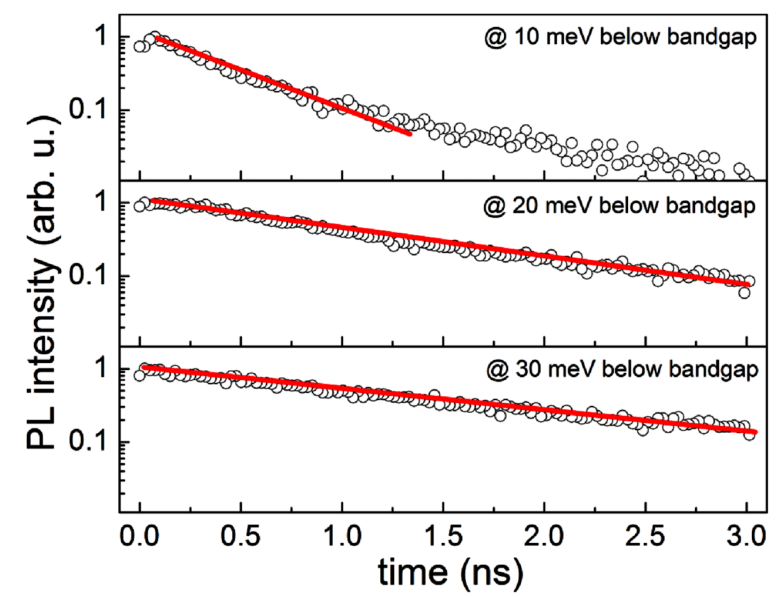

Fig. 1. Exemplary decay curves for different energies below the mobility edge, $\frac{N \alpha^{2}}{L^{2}}=0.4, \varepsilon_{0}=10 \mathrm{meV}$.

The $\tau_{\text {PL }}$ parameter determined from these fits for various energies and different simulation parameters are presented in Fig. 2a-d together with the PL spectrum integrated over the whole decay time. Figure $2 \mathrm{a}-\mathrm{d}$ shows the influence of localizing states density $\frac{N \alpha^{2}}{L^{2}}$ on decay 
time dispersion. We can see that the increase of $\frac{N \alpha^{2}}{L^{2}}$ causes two effects: (i) an increase of the PL full width at half maximum and (ii) an increase of decay times at low energy side and a decrease at high energy side which is better visible in Fig. 2e. However, to observe those changes the density of the localizing states has to change in the range of a few orders of magnitude. For example the increase of $\frac{N \alpha^{2}}{L^{2}}$ by one thousand times causes the increase of FWHM by about $50 \%$. The changes of the decay time constant for different energies below bandgap are connected with the rise of the hopping probability with increasing $\frac{N \alpha^{2}}{L^{2}}$.
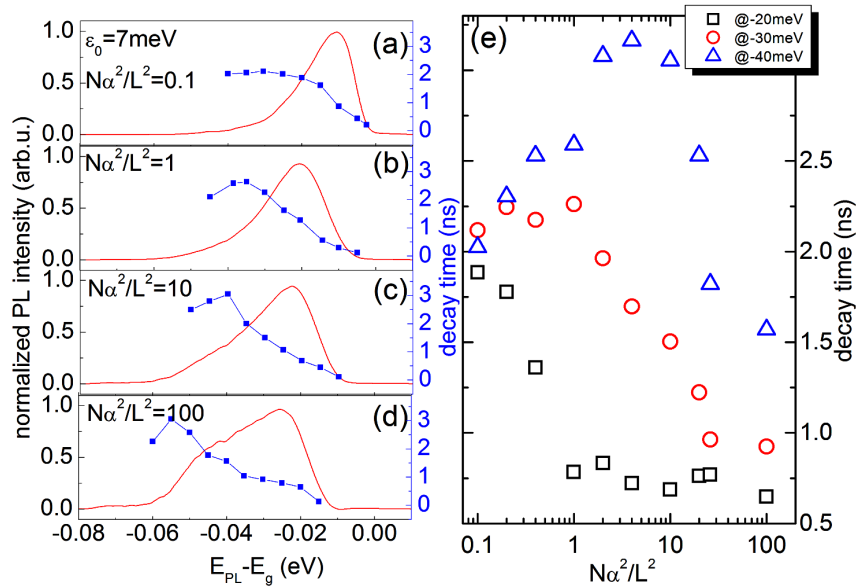

Fig. 2. (a)-(d) PL decay time dispersion for different values of $\frac{N \alpha^{2}}{L^{2}}$ together with the time integrated $\mathrm{PL}$ spectra, (e) decay time vs. $\frac{N \alpha^{2}}{L^{2}}$ for different energies.

Because the energy distribution of localizing states is exponential excitons are mostly captured by the shallow states. When the hopping probability is small then excitons do not perform too many hopping processes before recombination and they are not able to reach deeply situated localizing states (i.e., at low temperature hopping occurs mainly to low energy states). With increasing hopping probability the deeper situated states are refilled more effectively and the decay time at low energy becomes longer, at the same time at high energy side this time becomes shorter. In Fig. 2e we can see that for energy $-20 \mathrm{meV}$ below the mobility edge the decay time rapidly decreases with increasing $\frac{N \alpha^{2}}{L^{2}}$. In the same time for energy $-30 \mathrm{meV}$ and $-40 \mathrm{meV}$ we can observe initial increase connected with the refilling of these states from higher lying states. With further rise of $\frac{N \alpha^{2}}{L^{2}}$ the decrease of decay time constant occurs also for those energies which means that the transfer from these states to deeper situated states becomes more effective.

Figure 3a-d shows how the decay time dispersion changes with increasing localizing states energy together with PL spectra integrated over the whole decay time. With increasing $\varepsilon_{0}$ the decay time dispersion becomes clearly "broader" and an increase of FWHM can be eas- ily seen (the increase of FWHM is proportional to $\varepsilon_{0}$ $[12,14])$. From Fig. 3e we can see that with increasing deepness of localizing states the decay times at given energy becomes shorter which can be explained accordingly: when the $\varepsilon_{0}$ parameter increases the ratio between the number of shallow and deep localizing states increases as well. It means that there are more deep states to which exciton can be transported via hopping process.

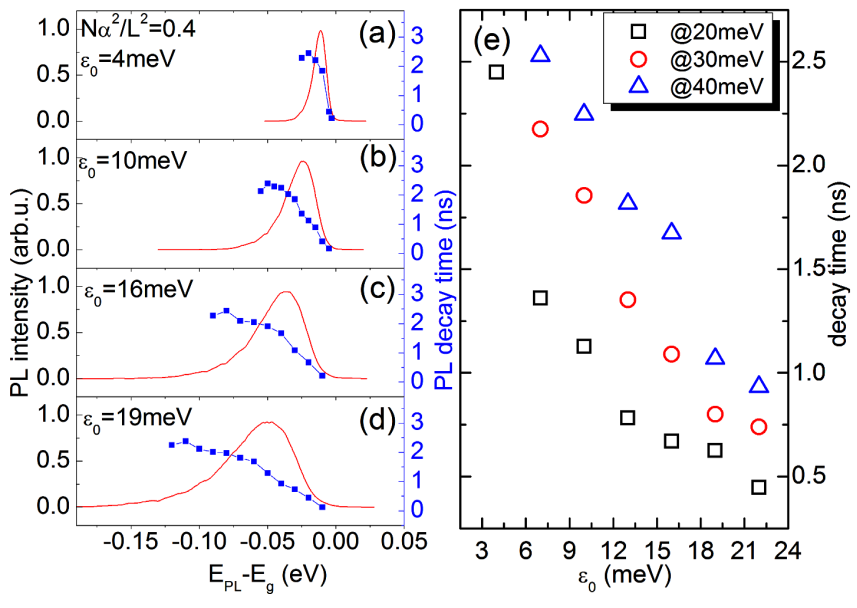

Fig. 3. (a)-(d) PL decay time dispersion for different values of $\varepsilon_{0}$ together with the time integrated PL spectra, (e) decay time vs. $\varepsilon_{0}$ for different energies.

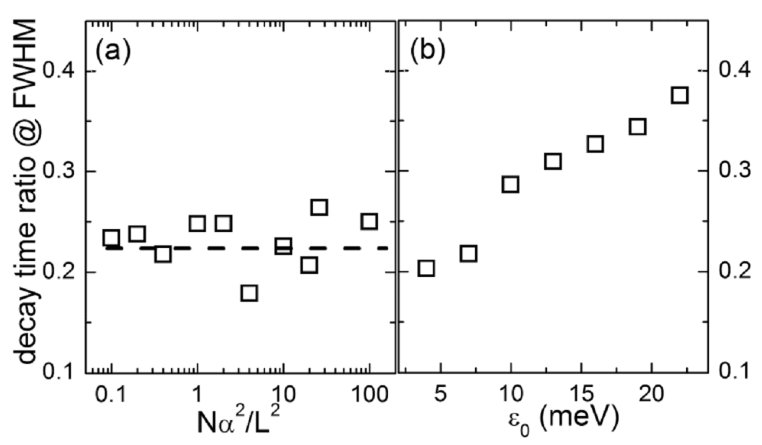

Fig. 4. Dependence of decay time ratio corresponding to half maximum of PL peak on (a) localizing state density $\frac{N \alpha^{2}}{L^{2}},(\mathrm{~b})$ average energy of localizing states $\varepsilon_{0}$.

The last thing that we want to present in this paper is the dependence of the decay time ratio taken at energies corresponding to half maximum of the PL peak. This ratio seems to be insensitive to the changes in the localizing state density, see Fig. 4. At the same time this ratio increases constantly with increasing $\varepsilon_{0}$. In our opinion this behavior can be applied to evaluate the influence of different technological processes (for example annealing conditions) on carrier localization in GaInNAs system. 


\section{Conclusion}

In conclusion, we have applied model of hopping excitons to investigate the influence of localizing state density and the average energy of localization on carrier dynamics in GaInNAs system. We believe that simulations presented here can be very helpful for experimental data analysis.

\section{Acknowledgments}

The authors acknowledge support from the Iuventus Plus program (IP2011 001471).

\section{References}

[1] M. Kondow, K. Uomi, A. Niwa, T. Kitatani, S. Watahiki, Y. Yazawa, Jpn. J. Appl. Phys. 35, 1273 (1996).

[2] A. Freundlich, A. Fotkatzikis, L. Bhusal, L. Williams, A. Alemu, W. Zhu, J.A.H. Coaquira, A. Feltrin, G. Radhakrishnan, J. Cryst. Growth 301-302, 993 (2007).

[3] I.A. Buyanova, W.M. Chen, G. Pozina, J.P. Bergman, B. Monemar, H.P. Xin, C.W. Tu, Appl. Phys. Lett. 75, 501 (1999).

[4] A. Kaschner, T. Lüttgert, H. Born, A. Hoffmann, A.Yu. Egorov, H. Riechert, Appl. Phys. Lett. 78, 1391 (2001).

[5] R.A. Mair, J.Y. Lin, H.X. Jiang, E.D. Jones, A.A. Allerman, S.R. Kurtz, Appl. Phys. Lett. 76, $188(2000)$.
[6] M. Latkowska, R. Kudrawiec, G. Sęk, J. Misiewicz, J. Ibáñez, M. Henini, M. Hopkinson, Phys. Status Solidi $C$ 8, 1655 (2011).

[7] M. Latkowska, R. Kudrawiec, G. Sęk, J. Misiewicz, J. Ibáñez, M. Henini, M. Hopkinson, Appl. Phys. Lett. 98, 131903 (2011).

[8] S.D. Baranovskii, R. Eichmann, P. Thomas, Phys. Rev. B 58, 13081 (1998).

[9] O. Rubel, S.D. Baranovskii, K. Hantke, B. Kunert, W.W. Rühle, P. Thomas, K. Volz, W. Stolz, Phys. Rev. B 73, 233201 (2006).

[10] M. Baranowski, M. Latkowska, R. Kudrawiec, J. Misiewicz, J. Phys., Condens. Matter 23, 205804 (2011).

[11] M. Baranowski, R. Kudrawiec, M. Latkowska, M. Syperek, J. Misiewicz, J.A. Gupta, Appl. Phys. Lett. 100, 202105 (2012).

[12] O. Rubel, M. Galluppi, S.D. Baranovskii, K. Volz, L. Geelhaar, H. Riechert, P. Thomas, W. Stolz, J. Appl. Phys. 98, 063518 (2005).

[13] M. Geddo, G. Guizzetti, M. Capizzi, A. Polimeni, D. Gollub, A. Forchel, Appl. Phys. Lett. 83, 470 (2003).

[14] M. Baranowski, M. Latkowska, R. Kudrawiec, J. Misiewicz, Acta Phys. Pol. A 120, 899 (2011). 